\title{
Super-resolution microscopy: going live and going fast
}

\author{
Melike Lakadamyali*[a]
}

Super-resolution microscopy is increasingly becoming an important tool for biological research, providing valuable information at the nanometer length scales inside cells and tissues. In the past decade numerous technological advancements have transformed superresolution microscopes into powerful tools of discovery. While the first super-resolution images took several hours to acquire, recent

\section{Introduction}

Over the centuries, light microscopy has become an enabling technology for all fields of biology. The non-invasive quality of visible light combined with the immense toolbox of fluorescent probes has allowed us to study cellular and sub-cellular biological processes, in real-time, in multiple colours, in 3D, inside living cells and even living animals. In the last decade, one of the major limitations of fluorescence microscopy, namely the diffraction limit, has also been overcome ${ }^{[1-5]}$, allowing researchers to generate multi-colour, 3D images of sub-cellular structures and protein nanodomains with unmatched spatial resolution. However, the long acquisition times that were reported for the first superresolution images ${ }^{[4]}$ originally limited the application of this powerful technology to fixed cells. In the recent years, thanks to the rapid pace of technological progress in this field, we have already started to see exciting dynamics at the nanoscale inside living cells obtained with super-resolution microscopes. However, live-cell super-resolution imaging is still in its early days and several parameters must be carefully weighed and balanced against each other to achieve the desired results. For example, ease of intracellular labelling, the brightness and photostability of fluorescent probes, their photoswitching kinetics, scanning speed of the focal spot, camera frame rates and phototoxicity are among several limiting factors that determine the final temporal and spatial resolution, field-of-view and imaging duration one can expect to achieve. This mini-review will highlight the recent technical advances that are enabling live-cell imaging with high resolution in space and in time. progress has led to tremendous improvement in acquisition speed, enabling researchers to probe dynamic processes in living cells with unprecedented spatiotemporal resolution. This mini-review focuses on the recent developments in live-cell super-resolution microscopy and its biological applications.

Melike Lakadamyali obtained her B.S. degree in Physics from the University of Texas at Austin in 2001. In 2006, she earned her Ph.D. degree in Physics at Harvard University under the supervision of Prof. Xiaowei Zhuang. After working as a postdoc in Prof. Jeff Lichtman's lab, she joined the Institut de Ciències Fotòniques (ICFO), Barcelona, Spain as a Group Leader and Nest Fellow. Dr.

Lakadamyali's research is in the area

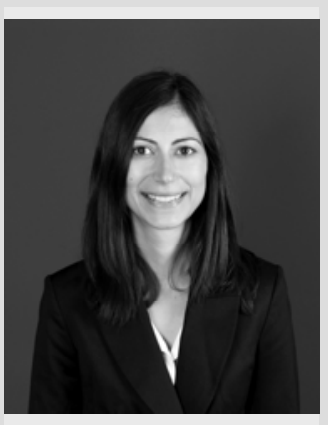
of single molecule biophysics and super-resolution fluorescence microscopy. She is currently interested in developing and applying advanced fluorescence microscopy methods to understand biological processes at the molecular scale.

\section{Live-cell super-resolution microscopy}

Super-resolution microscopy methods can be broadly divided into two categories: those that are based on patterning the illumination light, such as (Saturated) Structured Illumination Microscopy - $(\mathrm{S}) \mathrm{SIM}^{[2,6]}$ and Stimulated Emission Depletion Microscopy (STED) ${ }^{[1]}$ or those that are based on single molecule detection and localization, such as Stochastic Optical Reconstruction Microscopy - STORM ${ }^{[3]}$ and (Fluorescence) Photoactivation Localization Microscopy - PALM and fPALM ${ }^{[4,5]}$.

[a] Dr., M.L., Lakadamyali ICFO-Institut de Ciències Fotòniques Mediterranean Technology Park - Av. Carl Friedrich Gauss 3, 08860, Castelldefels (Barcelona), Spain Fax: (+)34 935534000

E-mail: melike.lakadamyali@icfo.es 
Live-cell imaging requires acquisition speed that is faster than the dynamics of the biological process to be studied. In addition, the ability to fluorescently label intracellular proteins with ease is highly important. Despite these technical challenges, live- cell super-resolution imaging has been demonstrated with almost all super-resolution microscopy methods with varying levels of spatiotemporal resolution. I summarize these recent developments below.

\section{(Saturated) Structured Illumination Microscopy - (S)SIM}

In (S)SIM patterned illumination light is used to achieve improved resolution ${ }^{[2,6]}$. Typically a sinusoidal pattern of bright and dark stripes is used for illumination by interfering two excitation beams. The patterned illumination helps shift the higher spatial frequency information in the sample to a range that can be imaged by conventional optics ${ }^{[6]}$. Typically, in the absence of saturation of fluorescence intensity, SIM leads to a 2 -fold improvement in spatial resolution, since the patterned light itself is diffraction limited ${ }^{[6,7]}$. Despite this modest improvement in spatial resolution, SIM is excellent for live-cell imaging applications, especially when a large field of view is required. The low illumination intensities used in SIM minimizes phototoxicity and its compatibility with a wide range of fluorescent probes enables easy intracellular labelling with genetically encoded fluorescent proteins. The imaging speed in SIM is limited by the speed with which the illumination pattern can be modulated and the camera speed ${ }^{[8]}$. A temporal resolution of $100 \mathrm{~ms}$ in $2 \mathrm{D}^{[9]}, 4 \mathrm{~s}$ in $3 \mathrm{D}^{[10]}$ and $8.5 \mathrm{~s}$ in multi-colour 3D ${ }^{[10]}$ imaging (Figure 1) has been achieved in living cells at the 2-fold enhanced spatial resolution. The achieved spatiotemporal resolution allowed observation of clathrin coated vesicle dynamics such as splitting and fusion events as well as clathrin mediated endocytosis (Figure 1). Further improvement of the spatial resolution in SIM requires much higher illumination light intensities (Saturated Structured Illumination Microscopy SSIM), such that the fluorescence emission scales non-linearly with excitation power ${ }^{[2]}$. So far, SSIM has not been demonstrated in living cells.

\section{Stimulated Emission Depletion - STED}

STED is the first super-resolution imaging method to be developed that is based on patterned illumination and is capable of breaking the diffraction limit ${ }^{[1]}$. STED uses a focused laser beam to excite fluorophores within a diffraction limited volume. Once excited, the fluorescence emission from a subset of these fluorophores can be supressed by forcing them back to the ground state through stimulated emission using a depletion beam $(\text { STED beam })^{[1]}$. The depletion beam is shaped to resemble a doughnut, which leads to an effective reduction in the excitation area to a sub-diffraction region in the centre of the doughnut ${ }^{[1]}$. Scanning the excitation and the STED beam across the sample generates a sub-diffraction image. The spatial resolution of the final image depends on the intensity of the STED beam ${ }^{[1]}$, and spatial resolution as high as $30 \mathrm{~nm}$ has been obtained in biological samples ${ }^{[11]}$.

The temporal resolution in STED is determined by the speed at which the focal spot can be scanned across the sample and the imaging area. Therefore, high temporal resolution can be achieved at the expense of field-of-view and/or spatial resolution. The dependence on field-of-view is due to the fact that it takes less time to image a smaller area at a given scanning speed. The compromise between spatial and temporal resolution is related to the scanning step size ${ }^{[8]}$. A larger step size leads to faster scanning at a reduced spatial resolution and vice versa ${ }^{[8]}$. Video rate STED imaging of synaptic vesicles has thus been achieved with $\sim 60 \mathrm{~nm}$ spatial resolution in a $5 \mu \mathrm{m}^{2}$ imaging area ${ }^{[12]}$. These studies showed, for the first time, the dynamics of individual synaptic vesicles (40 $\mathrm{nm}$ in diameter) inside the synaptic button and the mobility of these synaptic vesicles could be analysed in great detail. Synaptic vesicles seemed to be transiently trapped in hot spots of low mobility and also move with a combination of directional and diffusive motion within the synaptic button. Similarly, ER dynamics could be observed with sub-diffraction resolution in PtK2 cells using a $25 \mu \mathrm{m}^{2}$ imaging area with 10 seconds recording time per image ${ }^{[13]}$. Activity dependent changes to dendritic spine morphology could be measured in much greater detail than what has previously been possible in organotypic hippocampal slice cultures with 40 seconds per image temporal resolution ${ }^{[14]}$. These studies revealed that the changes in shapes of dendritic spines usually evolve from smaller and amorphous structures toward larger and more differentiated ones, often taking on cup-like shapes. These structural changes would have been very difficult to observe with conventional light microscopy.

To image deep inside tissues, STED has also been combined with two-photon excitation, generating sub-diffraction images of neuronal morphology $30 \mu \mathrm{m}$ deep in living brain tissue with $60 \mathrm{~nm}$ spatial resolution ${ }^{[15]}$. The use of continuous wave (CW) instead of pulsed lasers has dramatically improved the scanning speed ${ }^{[16]}$ enabling fast $(0.2$ seconds per frame) subdiffraction imaging in much larger areas $\left(\sim 70 \mu \mathrm{m}^{2}\right)^{[17]}$. In an exciting recent development, to further improve the compromise between temporal resolution and field of view, Chmyrov and colleagues have replaced the single doughnut illumination scheme with 100,000 doughnuts to scan the image simultaneously in a parallelized fashion ${ }^{[18]}$. This parallel detection scheme has enabled an impressive imaging speed of $<1$ second in very large fields-of-view $(120 \mu \mathrm{m} \times 100 \mu \mathrm{m})^{[18]}$.

The compatibility of STED with a wide range of fluorescent probes leads to flexibility in intracellular labelling for live-cell imaging. However, relatively high laser powers required for the STED beam means that photobleaching and phototoxicity can become a potential problem and limit the imaging duration as well as the overall choice of fluorophores to those that are bright and photostable (e.g. organic fluorophores such as Atto dyes). Despite these potential complications, repeated STED imaging of neurons in the cerebral cortex of a living mouse has recently been demonstrated ${ }^{[19]}$.

Stimulated emission is not the only saturable optical transition that can be exploited for super-resolution imaging with focused light. The STED concept has been extended to other optical transitions and this more general approach is referred to as reversible saturable optical fluorescence transition (RESOLFT) microscopy ${ }^{[20,21]}$. In RESOLFT, if fluorescent probes are switched off from long-lived states compared to the short excited lifetime exploited in STED, the intensity requirement for the depletion beam becomes much lower ${ }^{[21]}$. In particular, development of fatigue resistant fluorescent proteins that can switch between bright and dark states over thousands of $\operatorname{cycles}^{[22,23]}$ has made live-cell imaging with focused light at very low light intensities possible. This approach in combination with the parallelized detection scheme is likely the best way to acquire fast subdiffraction images of cellular structures for long time periods without inducing much photobleaching or phototoxicity using focused light methods.

Extremely fast detection in living cells has been achieved by combining fluorescence correlation spectroscopy (FCS) and STED ${ }^{[24]}$. FCS gives detailed information on the movement of 
molecules passing through an illumination spot by recording the fluctuations in fluorescence intensity ${ }^{[25]}$. STED-FCS decreases the focal volume through which the molecules must diffuse therefore enabling detection of dynamics at small length scales with millisecond temporal resolution ${ }^{[24]}$. While this approach does not allow sub-diffraction imaging of cellular structures, it is a powerful method for studying the mobility of small biomolecules within cells. For example, Eggeling and colleagues used STEDFCS to measure the mobility of different biomolecules (phosphoglycerolipids, sphingolipids

and glycosylphosphatidylinositol-anchored proteins) on the cell membrane. The superior resolution allowed them to observe the transient ( $10-20 \mathrm{~ms})$ trapping of phingolipids and glycosylphosphatidylinositol-anchored proteins, which dwelled within $<20-\mathrm{nm}$ diameter areas. They concluded that this trapping was due to the transient formations of cholesterol-assisted molecular complexes, such as lipid-protein binding or lipid shells.

\section{Localization based methods - STORM/PALM/fPALM}

Methods such as STORM/PALM and FPALM ${ }^{[3-5]}$ take advantage of the concept of single molecule detection and localization to break the diffraction limit. The position of a single fluorescent probe can be localized with very high precision (nanometer) determined mainly by the number of photons emitted by that probe $^{[26,27]}$. Single molecule localization is a powerful concept that has allowed precise tracking of the position and movement of individual proteins or organelles using single particle tracking ${ }^{[27-29]}$. This concept could be extended to super-resolution imaging of fluorescently labelled biological samples thanks to the discovery of photoswitchable fluorescent probes $\left.{ }^{[30}, 31\right]$. Photoswitching enables separation of many overlapping single molecule images in time. Using laser excitation, most of the fluoropores are put into a long-lived dark state ${ }^{[8]}$. Only a small subset of these fluorophores is stochastically activated into the fluorescent state by excitation with another wavelength of light (often UV illumination $)^{[8]}$. As a result, the single molecule images of this small subset of molecules do not overlap and their positions can thus be determined precisely. By repeating the process of activation, imaging and de-activation for several cycles, a superresolution image can be computed and reconstructed from molecule positions ${ }^{[8]}$. Localization based methods, therefore, require the use of fluorescent probes that can be photoswitched between bright and dark states. Today, there is a wide choice of these fluorescent probes, ranging from photoactivatable, photoconvertible or photoswitchable fluorescent proteins to photoswitchable organic fluorophores ${ }^{[32,33]}$ and progress in this field critically depends on the further development of such photoswitchable probes. In particular, new photoswitchable fluorescent proteins that are generated either through random mutation of the existing ones or through rational design are providing a lot of options for multi-colour, live-cell super-resolution imaging [22, 30, 34-41].

Spatial resolution in localization microscopy depends on several factors. One factor is the precision with which each molecule can be localized, which in turn mainly depends on the brightness of the molecule ${ }^{[26]}$. In addition, the label density limits the resolution due to the Nyquist criterion: the separation between neighbouring localizations must be one half of the desired resolution ${ }^{[42]}$. Finally the size of the probe used for tagging also plays a role ${ }^{[8]}$. In live-cell applications, much like in the case of STED, there is a trade-off between temporal and spatial resolution ${ }^{[8,42]}$. In this case, the temporal resolution is limited by the time needed to acquire enough localizations to satisfy the
Nyquist criterion for a given spatial resolution ${ }^{[8,42]}$. The temporal resolution is thus ultimately limited by the switching kinetics of the fluorophore, the camera frame rate and the field-of-view ${ }^{[8,42]}$.

Live-cell imaging with localization microscopy has been demonstrated with a wide range of probes. Fluorescent proteins provide easy intracellular labelling in living cells; however, the slow switching kinetics of fluorescent proteins and the low photon output limit both the spatial and the temporal resolution. Nevertheless, a temporal resolution of tens of seconds at $60-70$ $\mathrm{nm}$ spatial resolution has been achieved with a wide range of fluorescent proteins. For example, live-cell super-resolution imaging with PA-GFP, a photoactivatable fluorescent protein, revealed the dynamics of naoscale $(40 \mathrm{~nm}$ or larger) influenza heamagglutinin (HA) clusters on the plasma membrane ${ }^{[43]}$. The ability to observe both the size distribution and the mobility of the $\mathrm{HA}$-clusters at nanometer length scales was highly important to rule out several models for membrane domain organization. Dronpa, a photoswitchable fluorescent protein, was fused to the toxins, $\theta$-toxin and lysenin, to generate probes suitable for livecell super-resolution imaging of cholesterol- and sphingomyelinenriched membrane domains ${ }^{[44]}$. These super-resolution images showed two-types of cholesterol-enriched microdomains, lineshaped ones with lengths of $\sim 150 \mathrm{~nm}$ and round ones with a radius of $\sim 120 \mathrm{~nm}$, whereas the sphingomyelin-enriched microdomains were mostly round with a radius of $\sim 120 \mathrm{~nm}$. These observations led to the conclusion that cholesterol- and sphingomyelin-enriched domains occupy different regions of the plasma membrane, providing important insights on membrane organization $^{[44]}$. Paxillin labelled with tdEos, a photoconvertible fluorescent protein, and imaged in living cells showed the migration of adhesion complexes towards the cell interior ${ }^{[42]}$. Livecell super-resolution imaging in bacterial cells using EYFP, which blinks at high laser powers providing the photoswitching needed for localization microscopy, revealed the dynamics of bacterial actin protein MreB, which forms filamentous structures ${ }^{[45]}$. Livecell super-resolution imaging of different forms of mEos2-LcK, a thyrosine kinase that is involved in $\mathrm{T}$ cell antigen receptor phosphorylation, showed a highly dynamic clustering of Lck at the nanoscale dependent on Lck conformational states ${ }^{[46]}$. These studies suggested that Lck conformational states, rather than association with lipid domains and protein networks, represent an intrinsic mechanism for the intermolecular organization of early $T$ cell signaling. Overall, single molecule localization microscopy with fluorescent proteins is very powerful for studying nanoscale dynamics of slowly evolving biological processes (on time scales of several seconds) in living cells.

Recently, Zanacchi et al combined localization based superresolution microscopy with selective plane illumination microscopy $(\mathrm{SPIM})^{[47]}$ in living cells. In SPIM, the sample is illuminated by a thin sheet of light along an optical path that is orthogonal to the detection axis to achieve optical sectioning ${ }^{[48]}$. A $3 \mathrm{D}$ image of the sample can be generated by scanning the light sheet and/or rotating the sample with respect to the light sheet. Using this approach in combination with single molecule detection and localization (Individual molecule localization-selective plane illumination microscopy, IML-SPIM) Zanacchi et al could image PAmCherry tagged histone proteins and connexin 43 up to 50$100 \mu \mathrm{m}$ deep inside living spheroids in three dimensions with a spatial resolution of $<60 \mathrm{~nm}^{[47]}$.

Organic fluorophores are typically brighter than fluorescent proteins and they can be switched to dark states very fast by using high laser powers without compromising photon output ${ }^{[49]}$. Therefore, using organic fluorophores and high enough laser power to switch the fluorophores off within one camera frame 
(camera frame rate was $500 \mathrm{~Hz}$ in this case), Jones and colleagues could reconstruct STORM images within 1-2 seconds while maintaining an impressive $30 \mathrm{~nm}$ lateral and $50 \mathrm{~nm}$ axial 3D spatial resolution ${ }^{[49]}$. Using live-cell 3D super-resolution imaging, they could observe the internalization of transferrin receptor through clathrin-coated pits. However, these improvements came at the expense of ease of intracellular labelling. Intracellular labelling with organic fluorophores in living cells can be achieved using genetically encoded tags such as SNAP or HaLo tag as previously demonstrated for live-cell super-resolution imaging of bacterial proteins or histone proteins in mammalian cells ${ }^{[50,51]}$. These tags react with a small peptide, which contains an organic fluorophore as a label. The organic fluorophore can thus be specifically targeted to a protein of choice. However, conjugation with certain fluorophores (in particular the most commonly used and brightest photoswitchable fluorophore Alexa 647) renders the peptide cell impermeable. Therefore, to target Alexa 647-labelled peptide to intracellular compartments, complex methods are needed such as electroporation or bead-loading, in which glass microbeads are sprinkled onto cells to temporarily disrupt the plasma membrane ${ }^{[49]}$.

The realization that many organic fluorophores are photoswitchable under the right buffer conditions ${ }^{[52]}$ led to the identification of several photoswitchable live-cell compatible membrane probes, allowing super-resolution imaging of many cellular organelles in living cells (ER, mitochondria, lysosomes or the cell membrane) ${ }^{[53]}$ (Figure 2). These time-lapse STORM images revealed thin, extended tubular intermediates connecting neighbouring mitochondria during mitochondrial fusion and fission. These tubular intermediates were obscured in conventional time lapse microscopy due to their small diameter $(\sim 100 \mathrm{~nm})$. In addition, nucleic acid binding dyes such as picogreen recently allowed imaging of DNA dynamics at the nanometer length scales $^{[54]}$.

Recent advances in data analysis methods for localization microscopy have led to further improvements in the temporal resolution. Early analysis methods required the single molecule images to be mostly non-overlapping to determine their positions accurately ${ }^{[3,4]}$. However, this requirement can be largely relaxed to allow the positions of highly overlapping molecules to be precisely determined by using data analysis methods such as multi-emitter fitting or sparse-signal recovery ${ }^{[55-59]}$. Therefore, image acquisition can be sped up since the Nyquist criterion can be satisfied more rapidly by activating several partially overlapping molecules simultaneously in each frame. However, it is important to note that while improving temporal resolution, multi-emitter fitting often leads to decreased spatial resolution. Nevertheless, using one of these algorithms, microtubule dynamics could be imaged inside living cells using mEos2 fluorescent protein with a temporal resolution of $3 \mathrm{~s}$ and spatial resolution of $60 \mathrm{~nm}^{[59]}$.

While the temporal resolution of super-resolution microscopy has seen a dramatic improvement from the early days, a combination of millisecond scale temporal resolution, large field-of-view and relatively long imaging duration (several minutes) has been challenging to achieve. Recently, conventional live-cell imaging and single particle tracking has been combined with localization based super-resolution microscopy in a sequential and correlated way ${ }^{[60]}$. This all-optical, correlative imaging approach has made it possible to interpret millisecond dynamics of organelle transport processes in the context of nanoscale $3 \mathrm{D}$ organization of the microtubule cytoskeleton ${ }^{[60]}$ (Figure 3). Therefore, the behaviour of motor-protein transported organelles could be observed at microtubule intersections. It was found that the axial separation of microtubules determines if a cargo can fit through and pass the intersection. While this correlative approach does not directly address the problem of improving temporal resolution in super-resolution microscopy, it circumvents this problem and increases the information content that can be obtained by correlating fast dynamics with superresolution images.

Huang and colleagues have recently achieved very impressive video-rate super-resolution imaging by adapting the image analysis methods used in localization microscopy to scientific complementary metal-oxide semiconductor (sCMOS) cameras ${ }^{[61]}$. SCMOS cameras combine the advantages of high quantum efficiency, large field-of-view and very fast readout speeds. However, sCMOS cameras suffer from highly pixeldependent noise characteristics making it difficult to estimate single molecule positions using the common algorithms that employ Poisson distributed, pixel-independent noise models ${ }^{[61]}$. Huang et al. developed new localization algorithms specially adapted to account for pixel-dependent noise in sCMOS cameras $^{[61]}$. Using organic fluorophores, they demonstrated subdiffraction images of transferrin clusters with a very impressive 31 $\mathrm{ms}$ temporal resolution and $13 \times 13 \mu \mathrm{m}^{2}$ field of view[61]. This approach combined with multi-emitter fitting algorithms and bright organic fluorophores provides the highest spatiotemporal resolution and the largest field of view in single molecule localization microscopy reported thus far. Future biological applications of sub-diffraction imaging using this approach will likely lead to exciting new discoveries.

Finally, photoactivation has also been used for high density single molecule tracking of tagged proteins inside living cells with millisecond temporal resolution ${ }^{[62]}$. This approach, referred to as single particle tracking PALM (spt-PALM), allows tracking the motion and dynamics of a much larger amount of target molecules than what is possible with conventional single molecule tracking approaches. Spt-PALM is a powerful approach for generating global diffusion maps ${ }^{[63]}$ and flow diagrams of biomolecules inside cells ${ }^{[64]}$. The single molecule trajectories can also often be explored to reconstruct the shape of the underlying structure ${ }^{[63]}$.

\section{Figures}
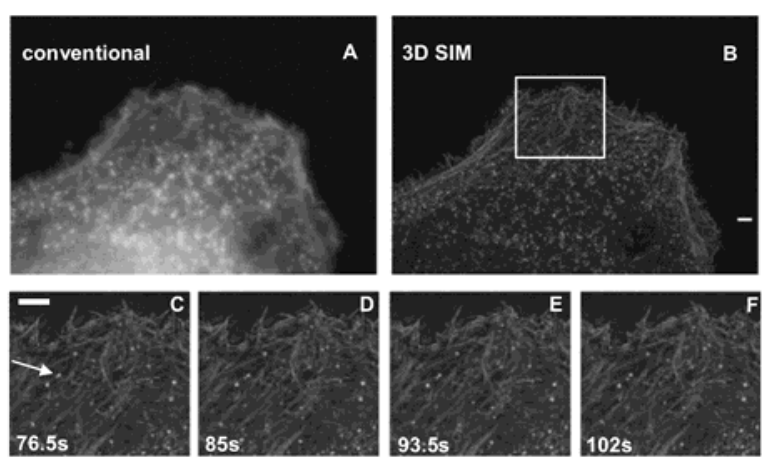

Figure 1. Live-cell 3D SIM imaging of clathrin coated pits (green) and actin cytoskeleton (red). The two-colour images were acquired with a temporal resolution of 8.5 seconds. A clathrin coated vesicle (arrow) can be seen splitting into two. Reproduced with permission from Fiolka et al., PNAS, 109, 5311-5315 (2012). 


\section{Outlook}
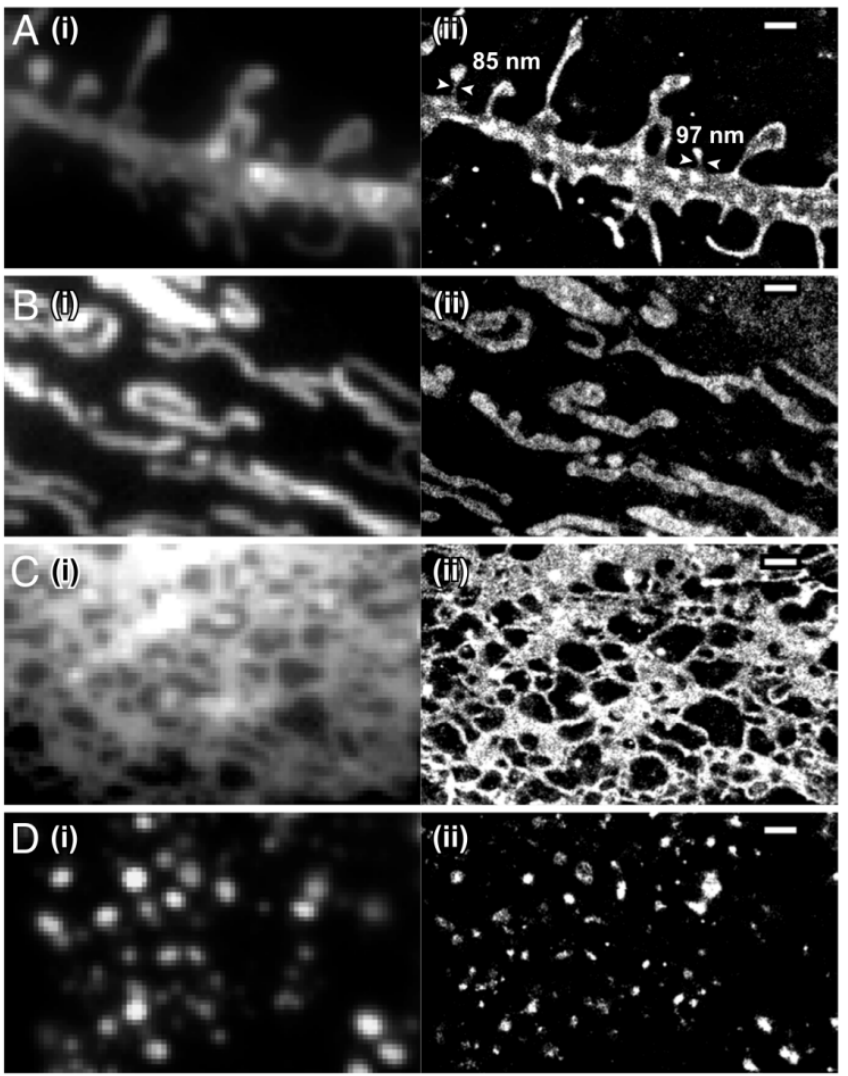

Figure 2. Live-cell STORM imaging of cellular organelles with membrane probes. (A) Cell membrane imaged with Dil in live neurons, (B) Mitochondria imaged with MitoTracker Red in BSC-1 cells, (C) Endoplasmic Reticulum (ER) imaged with ER-Tracker Red in BSC-1 cells and (D) Lysosomes imaged with LysoTracker Red in BSC-1 cells. Reproduced with permission from Shim et al., PNAS, 109, 13978-13983 (2012).

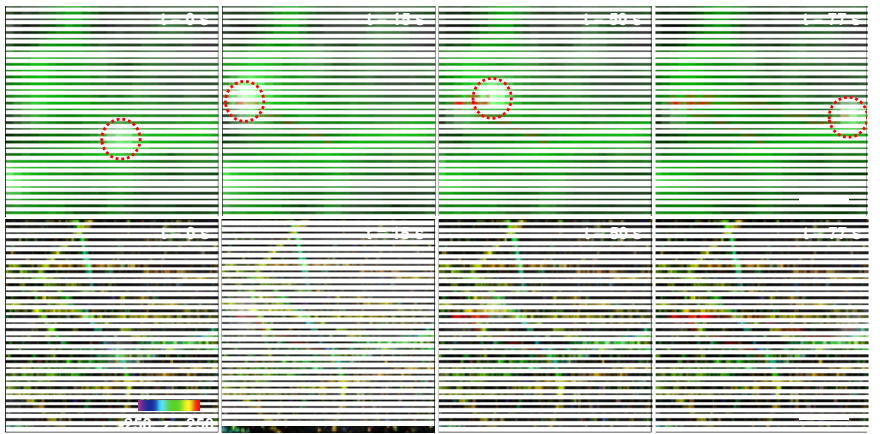

Figure 3. Correlative live-cell and STORM imaging of cargo transport. (Above) Conventional two-colour time lapse images of lysosome (white) and microtubules (green). The red line shows the transport trajectory of the lysosome obtained with single particle tracking. (Below) The same field-of-view but with the conventional image of microtubules replaced by the 3D STROM image (colour coding shows z-scale according to the z-colour bar). The transport trajectory of lysosome could be mapped precisely to the STORM image of individual microtubules. Scale bar $500 \mathrm{~nm}$. For more details, see reference: Balint et al., PNAS, 110, 3375-3380 (2013)
The ability to non-invasively image dynamic processes in living cells is one of the greatest advantages of light microscopy. Combining this capability with sub-diffraction spatial resolution holds great promise for new discoveries. The rapid development in super-resolution fluorescence microscopy that followed its first introduction about a decade ago has enabled researchers to image sub-cellular structures and protein nanodomains with unmatched spatiotemporal resolution with all the existing superresolution microscopy methods. The exact method of choice depends on the biological application, in particular, the spatiotemporal resolution, field-of-view and imaging length that is needed. These parameters must be carefully considered and balanced to guide the choice of a particular method. SIM provides large field of view at high temporal resolution, easy and flexible labelling options and low light intensities but the spatial resolution is modest $(\sim 100 \mathrm{~nm})$. For higher spatial resolution $(50-70 \mathrm{~nm})$ and imaging speeds in the order of few seconds to tens of seconds without compromising the flexible intracellular labelling, CWSTED or single molecule localization methods with fluorescent proteins provide a good option. Further improvement in speed and field of view can be achieved via single molecule localization with organic flurophores using sCMOS camera detection and multi-emitter fitting algorithms. Alternatively, STED or RESOLFT combined with parallel detection using many doughnuts can provide high spatiotemporal resolution and large field of view. When imaging in thick samples the various methods can be combined with two-photon excitation or selective plane illumination.

As the impressive pace of development in this field continues, we should expect to be able to observe highly dynamic processes at the nanometer scale inside living cells, tissues and even animals, leading to breakthrough discoveries in cell and molecular biology. Further improvement of spatial and temporal resolution will require development of new fatigue resistant probes with faster photoswitching kinetics, higher photon output and higher photostability. With the active search for better fluorescent probes under way, the coming years will surely see exciting new developments in the field of photoswitchable fluorescent proteins and photoswitchable fluorophores with improved photophysical properties, opening new doors in the field of live-cell super-resolution microscopy.

\section{Acknowledgements}

This work was supported in part by Fundació Cellex, Barcelona, Marie-Curie International Reintegration Grant FP7-PEOPLE2010-RG and the Plan Nacional Grant from the Spanish Ministry (Ministerio de Economia y Competitividad) FIS2012-37753.

Keywords: super-resolution microscopy · live-cell imaging STORM $\cdot$ PALM $\cdot$ STED $\cdot$ SIM

[1] T. A. Klar, S. Jakobs, M. Dyba, A. Egner, S. W. Hell Proceedings of the National Academy of Sciences of the United States of America. 2000, 97, 8206-8210

[2] M. G. Gustafsson Proceedings of the National Academy of Sciences of the United States of America. 2005, 102, 13081-13086. 
[3] M. J. Rust, M. Bates, X. Zhuang Nature methods. 2006, 3, 793-795.

[4] E. Betzig, G. H. Patterson, R. Sougrat, O. W. Lindwasser, S. Olenych, J. S. Bonifacino, M. W. Davidson, J. Lippincott-Schwartz, H. F. Hess Science. 2006, 313, 1642-1645.

[5] S. T. Hess, T. P. Girirajan, M. D. Mason Biophysical journal. 2006, 91, 4258-4272.

[6] M. G. Gustafsson Journal of microscopy. 2000, 198, 82-87.

[7] M. G. Gustafsson, L. Shao, P. M. Carlton, C. J. Wang, I. N. Golubovskaya, W. Z. Cande, D. A. Agard, J. W. Sedat Biophysical journal. 2008, 94, 4957-4970.

[8] B. Huang, H. Babcock, X. Zhuang Cell. 2010, 143, 1047-1058.

[9] P. Kner, B. B. Chhun, E. R. Griffis, L. Winoto, M. G. Gustafsson Nature methods. 2009, 6, 339-342.

[10] R. Fiolka, L. Shao, E. H. Rego, M. W. Davidson, M. G. Gustafsson Proceedings of the National Academy of Sciences of the United States of America. 2012, 109, 5311-5315.

[11] R. Schmidt, C. A. Wurm, A. Punge, A. Egner, S. Jakobs, S. W. Hell Nano letters. 2009, 9, 2508-2510.

[12] V. Westphal, S. O. Rizzoli, M. A. Lauterbach, D. Kamin, R. Jahn, S. W. Hell Science. 2008, 320, 246-249.

[13] B. Hein, K. I. Willig, S. W. Hell Proceedings of the National Academy of Sciences of the United States of America. 2008, 105, 14271-14276.

[14] U. V. Nagerl, K. I. Willig, B. Hein, S. W. Hell, T. Bonhoeffer Proceedings of the National Academy of Sciences of the United States of America. 2008, 105, 18982-18987.

[15] K. T. Takasaki, J. B. Ding, B. L. Sabatini Biophysical journal. 2013, 104, 770-777.

[16] K. I. Willig, B. Harke, R. Medda, S. W. Hell Nature methods. 2007, 4, 915-918

[17] G. Moneron, R. Medda, B. Hein, A. Giske, V. Westphal, S. W. Hell Optics express. 2010, 18, 1302-1309.

[18] A. Chmyrov, J. Keller, T. Grotjohann, M. Ratz, E. d'Este, S. Jakobs, C. Eggeling, S. W. Hell Nature methods. 2013.

[19] S. Berning, K. I. Willig, H. Steffens, P. Dibaj, S. W. Hell Science. 2012, $335,551$.

[20] S. W. Hell Nature biotechnology. 2003, 21, 1347-1355.

[21] M. Hofmann, C. Eggeling, S. Jakobs, S. W. Hell Proceedings of the National Academy of Sciences of the United States of America. 2005, 102, 17565-17569.

[22] T. Brakemann, A. C. Stiel, G. Weber, M. Andresen, I. Testa, T. Grotjohann, M. Leutenegger, U. Plessmann, H. Urlaub, C. Eggeling, M. C. Wahl, S. W. Hell, S. Jakobs Nature biotechnology. 2011, 29, 942-947.

[23] T. Grotjohann, I. Testa, M. Leutenegger, H. Bock, N. T. Urban, F. Lavoie-Cardinal, K. I. Willig, C. Eggeling, S. Jakobs, S. W. Hell Nature. 2011, 478, 204-208.

[24] C. Eggeling, C. Ringemann, R. Medda, G. Schwarzmann, K. Sandhoff, S. Polyakova, V. N. Belov, B. Hein, C. von Middendorff, A. Schonle, S. W. Hell Nature. 2009, 457, 1159-1162.

[25] E. Haustein, P. Schwille Annual review of biophysics and biomolecular structure. 2007, 36, 151-169.

[26] R. E. Thompson, D. R. Larson, W. W. Webb Biophysical journal. 2002, 82, 2775-2783.

[27] A. Yildiz, J. N. Forkey, S. A. McKinney, T. Ha, Y. E. Goldman, P. R. Selvin Science. 2003, 300, 2061-2065.

[28] D. Cai, D. P. McEwen, J. R. Martens, E. Meyhofer, K. J. Verhey PLoS biology. 2009, 7, e1000216.
[29] M. J. Rust, M. Lakadamyali, B. Brandenburg, X. Zhuang Cold Spring Harbor protocols. 2011, 2011.

[30] G. H. Patterson, J. Lippincott-Schwartz Science. 2002, 297, 18731877.

[31] M. Bates, T. R. Blosser, X. Zhuang Physical review letters. 2005, 94, 108101.

[32] M. Fernandez-Suarez, A. Y. Ting Nature reviews. Molecular cell biology. 2008, 9, 929-943.

[33] J. Lippincott-Schwartz, G. H. Patterson Trends in cell biology. 2009, 19, 555-565.

[34] N. G. Gurskaya, V. V. Verkhusha, A. S. Shcheglov, D. B. Staroverov, T. V. Chepurnykh, A. F. Fradkov, S. Lukyanov, K. A. Lukyanov Nature biotechnology. 2006, 24, 461-465.

[35] S. Habuchi, R. Ando, P. Dedecker, W. Verheijen, H. Mizuno, A. Miyawaki, J. Hofkens Proceedings of the National Academy of Sciences of the United States of America. 2005, 102, 9511-9516.

[36] S. Habuchi, H. Tsutsui, A. B. Kochaniak, A. Miyawaki, A. M. van Oijen PloS one. 2008, 3, e3944.

[37] A. L. McEvoy, H. Hoi, M. Bates, E. Platonova, P. J. Cranfill, M. A. Baird, M. W. Davidson, H. Ewers, J. Liphardt, R. E. Campbell PloS one. 2012, 7, e51314.

[38] S. A. McKinney, C. S. Murphy, K. L. Hazelwood, M. W. Davidson, L. L. Looger Nature methods. 2009, 6, 131-133.

[39] F. V. Subach, G. H. Patterson, S. Manley, J. M. Gillette, J. Lippincott-Schwartz, V. V. Verkhusha Nature methods. 2009, 6 , 153-159.

[40] M. Zhang, H. Chang, Y. Zhang, J. Yu, L. Wu, W. Ji, J. Chen, B. Liu, J. Lu, Y. Liu, J. Zhang, P. Xu, T. Xu Nature methods. 2012, 9, 727729.

[41] R. Ando, C. Flors, H. Mizuno, J. Hofkens, A. Miyawaki Biophysical journal. 2007, 92, L97-99.

[42] H. Shroff, C. G. Galbraith, J. A. Galbraith, E. Betzig Nature methods. 2008, 5, 417-423.

[43] S. T. Hess, T. J. Gould, M. V. Gudheti, S. A. Maas, K. D. Mills, J. Zimmerberg Proceedings of the National Academy of Sciences of the United States of America. 2007, 104, 17370-17375.

[44] H. Mizuno, A. Mitsuhiro, P. Dedecker, A. Makino, S. Rocha, Y. Ohno-Iwashita, J. Hofkens, T. Kobayashi, A. Miyawaki Chemical Science. 2011, 2

[45] J. S. Biteen, M. A. Thompson, N. K. Tselentis, G. R. Bowman, L. Shapiro, W. E. Moerner Nature methods. 2008, 5, 947-949.

[46] J. Rossy, D. M. Owen, D. J. Williamson, Z. Yang, K. Gaus Nature immunology. 2013, 14, 82-89.

[47] F. Cella Zanacchi, Z. Lavagnino, M. Perrone Donnorso, A. Del Bue, L. Furia, M. Faretta, A. Diaspro Nature methods. 2011, 8, 10471049.

[48] J. Huisken, J. Swoger, F. Del Bene, J. Wittbrodt, E. H. Stelzer Science. 2004, 305, 1007-1009.

[49] S. A. Jones, S. H. Shim, J. He, X. Zhuang Nature methods. 2011, 8, 499-508.

[50] H. L. Lee, S. J. Lord, S. Iwanaga, K. Zhan, H. Xie, J. C. Williams, H. Wang, G. R. Bowman, E. D. Goley, L. Shapiro, R. J. Twieg, J. Rao, W. E. Moerner Journal of the American Chemical Society. 2010, 132, 15099-15101.

[51] R. Wombacher, M. Heidbreder, S. van de Linde, M. P. Sheetz, M. Heilemann, V. W. Cornish, M. Sauer Nature methods. 2010, 7, 717719.

[52] M. Heilemann, S. van de Linde, A. Mukherjee, M. Sauer Angewandte Chemie. 2009, 48, 6903-6908. 
[53] S. H. Shim, C. Xia, G. Zhong, H. P. Babcock, J. C. Vaughan, B. Huang, X. Wang, C. Xu, G. Q. Bi, X. Zhuang Proceedings of the National Academy of Sciences of the United States of America. 2012, 109, 13978-13983.

[54] A. Benke, S. Manley Chembiochem : a European journal of chemical biology. 2012, 13, 298-301.

[55] F. Huang, S. L. Schwartz, J. M. Byars, K. A. Lidke Biomedical optics express. 2011, 2, 1377-1393.

[56] S. J. Holden, S. Uphoff, A. N. Kapanidis Nature methods. 2011, 8, 279-280.

[57] H. Babcock, Y. M. Sigal, X. Zhuang Optical Nanoscopy. 2012, 1:6.

[58] S. Cox, E. Rosten, J. Monypenny, T. Jovanovic-Talisman, D. T. Burnette, J. Lippincott-Schwartz, G. E. Jones, R. Heintzmann Nature methods. 2012, 9, 195-200.

[59] L. Zhu, W. Zhang, D. Elnatan, B. Huang Nature methods. 2012, 9, 721-723.

[60] S. Balint, I. Verdeny Vilanova, A. Sandoval Alvarez, M. Lakadamyali Proceedings of the National Academy of Sciences of the United States of America. 2013, 110, 3375-3380.
[61] F. Huang, T. M. Hartwich, F. E. Rivera-Molina, Y. Lin, W. C. Duim, J. J. Long, P. D. Uchil, J. R. Myers, M. A. Baird, W. Mothes, M. W. Davidson, D. Toomre, J. Bewersdorf Nature methods. 2013, 10, 653-658.

[62] S. Manley, J. M. Gillette, G. H. Patterson, H. Shroff, H. F. Hess, E. Betzig, J. Lippincott-Schwartz Nature methods. 2008, 5, 155-157.

[63] N. Hoze, D. Nair, E. Hosy, C. Sieben, S. Manley, A. Herrmann, J. B. Sibarita, D. Choquet, D. Holcman Proceedings of the National Academy of Sciences of the United States of America. 2012, 109, 17052-17057.

[64] D. T. Burnette, S. Manley, P. Sengupta, R. Sougrat, M. W. Davidson, B. Kachar, J. Lippincott-Schwartz Nature cell biology. 2011, 13, 371-381.

Received: ((will be filled in by the editorial staff)) Published online: ((will be filled in by the editorial staff)) 
Entry for the Table of Contents (Please choose one layout)

\section{MINIREVIEWS}

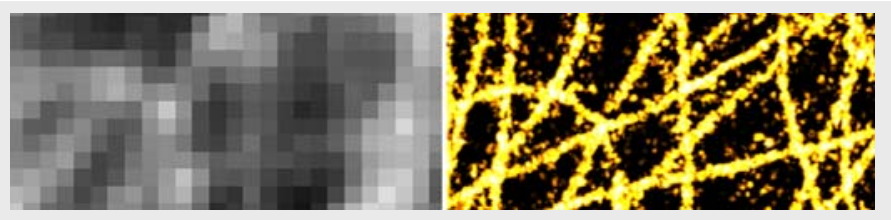

Far-field fluorescence microscopy has undergone a revolution with the development of super-resolution microscopes. In less than ten years, these microscopes are already peering into biological processes in living cells with unmatched spatiotemporal resolution and bringing about exciting new discoveries in biology. The rapid pace of development promises that live-cell super-resolution microscopy will soon become the indispensable tool for every biologist.
Melike Lakadamyali*

Page No. - Page No.

Super-resolution microscopy: going live and going fast 